\title{
PARÂMETROS FITOSSOCIOLÓGICOS DE UM CERRADO NO PARQUE NACIONAL DA SERRA DO CIPÓ, MG ${ }^{1}$
}

\author{
João Augusto Alves Meira Neto² e Amilcar Walter Saporetti Júnior ${ }^{3}$
}

\begin{abstract}
RESUMO - A riqueza de espécies e os fatores determinantes da ocorrência da vegetação de cerrado há muito têm despertado o interesse de pesquisadores. Muitos cerrados protegidos em unidades de conservação ainda não foram investigados florística e estruturalmente. Neste trabalho foi realizado um levantamento florístico e

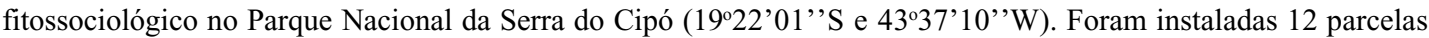
de $150 \mathrm{~m}^{2}$ e foram amostrados todos os indíviduos lenhosos com circunferência do caule à altura do solo maior ou igual a $10 \mathrm{~cm}$. Foram relacionadas 44 espécies de 37 gêneros e 30 famílias. Entre estas, Leguminosae Caesalpinioideae, Leguminosae Mimosoideae e Guttiferae, com três espécies cada, foram as mais ricas. As espécies mais importantes (VI) foram Hymenaea stigonocarpa, Allagoptera campestris, Diospyros hispida, Rapanea guianensis e Piptocarpha rotundifolia.
\end{abstract}

Palavras-chave: Fitossociologia, Serra do Cipó, cerrado e savana.

\section{PHYTOSOCIOLOGICAL PARAMETERS OF A CERRADO IN “SERRA DO CIPÓ” NATIONAL PARK, MINAS GERAIS, BRAZIL}

\begin{abstract}
The diversity of species and the factors determining the occurrence of cerrado vegetation have long called the attention of researchers. Many protected cerrados in Conservation Units have not been floristically and structurally studied. This work conducted a floristic and phytosociological survey of the Parque Nacional da

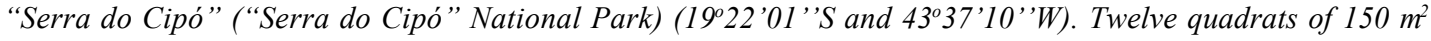
were established and all individuals with a stem circumference at ground level larger than or equal to $10 \mathrm{~cm}$ were sampled. A total of 44 species of 37 genera and 30 families were found. The richest families were Leguminosae Caesalpinioideae, Leguminosae Mimosoideae and Guttiferae, with three species each. The most important species (VI) were Hymenaea stigonocarpa, Allagoptera campestris, Diospyros hispida, Rapanea guianensis and Piptocarpha rotundifolia.
\end{abstract}

Key words: $\quad$ Phytosociology, "Serra do Cipó” National Park, cerrado and savanna.

\section{INTRODUÇÃO}

O cerrado, caracterizado como uma vegetação de savana na classificação internacional, localiza-se predominantemente no Planalto Central do Brasil e constitui a segunda maior formação vegetal brasileira em extensão, sendo a primeira a Floresta Amazônica. Esse bioma se estende por cerca de 2 milhões de quilômetros quadrados, representando $22 \%$ do território nacional (Ratter, 1992).
Cole (1960) e Eiten (1972), entre muitos outros autores, afirmaram que fatores edáficos, principalmente os teores de nutrientes, além do fogo e da intervenção humana, determinam as diferentes fitofisionomias da vegetação do cerrado.

Os levantamentos florísticos já realizados mostraram a grande riqueza de espécies e que em toda extensão do cerrado ocorre uma contínua variação na composição florística (Ratter et al., 2000). Ainda são inúmeras as áreas em unidades de conservação com vegetação de cerrado

1 Recebido para publicação em 10.9.2001.

Aceito para publicação em 6.11.2002.

Trabalho realizado na excursão para a Serra do Cipó da disciplina de BIO-330, Ecologia Geral.

2 Departamento de Biologia Vegetal da Universidade Federal de Viçosa - DBV/UFV - 36571-000 Viçosa-MG, $<$ j.meira@mail.ufv.br>; ${ }^{3}$ Estagiário do Departamento de Biologia Vegetal da UFV, Graduação em Ciências Biológicas/UFV. 
sem informações a respeito de sua composição e estrutura. O objetivo deste trabalho foi realizar um estudo fitossociológico de uma área de cerrado sensu stricto no Parque Nacional da Serra do Cipó-MG.

O parque localiza-se na Serra do Cipó, uma das unidades da Serra do Espinhaço, em área que pertence aos municípios de Morro do Pilar, Baldim, Jaboticatubas e Conceição do Mato Dentro. A área de estudos localizase no município de Jaboticatubas, nas coordenadas $19^{\circ} 22^{\prime} 01^{\prime}$ 'S e $43^{\circ} 37^{\prime} 10^{\prime}$ 'W, a uma altitude de $850 \mathrm{~m}$. Geomorfologicamente, o local está num pediplano onde o tipo de solo é Latossolo Vermelho. A precipitação média anual é de cerca de $1.500 \mathrm{~mm}$ e o clima é Cwb pelo sistema de Köppen, com quatro a cinco meses secos. Dentre os impactos ambientais na área pode-se citar a ocorrência da passagem de fogo, em 1999.

O trabalho de campo foi feito no segundo semestre de 2000. O método fitossociológico aplicado foi o de parcelas (Mueller-Dombois \& Ellenberg, 1974). Foram instaladas 12 parcelas de $10 \times 15 \mathrm{~m}$ em cerrado sensu stricto, perfazendo uma área amostral de 0,18 ha. Foram amostrados todos os indivíduos com $10 \mathrm{~cm}$ ou mais de circunferência do caule no nível do solo (CAS). Os indivíduos da espécie de palmeira-acaule (Allagoptera campestris), por apresentarem grande presença, foram incluídos na amostragem, tendo sido medida a circunferência da roseta de folhas à altura do solo.

A identificação taxonômica foi efetuada mediante consultas a herbários, consultas a especialistas e por meio da literatura especializada.

O sistema de classificação utilizado foi o de Cronquist (1998).Todos os nomes de espécies e suas respectivas autoridades foram confirmados e atualizados pelo "software" do índice de espécies do ROYAL BOTANIC GARDENS OF KEW (1993) ou mediante consulta à literatura com informações mais atualizadas que as desse programa.

Pelo levantamento realizado foram amostradas 44 espécies lenhosas, distribuídas em 37 gêneros e 29 famílias. As famílias com maior número de espécies foram Leguminosae Caesalpinioideae, Guttiferae e Leguminosae Mimosoideae, com três espécies cada, seguidas de Sapotaceae, Asteraceae, Erythroxylaceae, Annonaceae, Nyctaginaceae, Leguminosae Faboideae, Myrtaceae, Rubiaceae e Vochysiaceae, com duas espécies (Quadro 1).

R. Árvore, Viçosa-MG, v.26, n.5, p.645-648, 2002
As espécies com maiores densidades relativas (DR) foram Hymenaea stigonocarpa (16,60\%), Allagoptera campestris (8,40\%), Diospyros hispida (7,46\%), Rapanea guianensis e Erythroxylum daphnites (5,78\%) e Piptocarpha rotundifolia $(5,22 \%)$. As espécies que apresentaram maiores freqüências relativas foram Diospyros hispida $(6,42 \%)$, Byrsonima verbascifolia (5,88\%), Hymenaea stigonocarpa, Allagoptera campestris, Piptocarpha rotundifolia, Aspidosperma tomentosum e Acosmium dasycarpum (5,35\%), Pouteria ramiflora e Kielmeyera coriacea (4,81\%), Rapanea guianensis e Erythroxylum daphnites $(4,28)$. As espécies que apresentaram maiores dominâncias relativas (DoR) foram Hymenaea stigonocarpa (23,24\%), Rapanea guianensis (8,08\%), Guapira noxia (6,09\%), Agonandra brasiliensis (5,73\%) e Annona coriacea (5,67\%). As espécies de maiores valores de importância (VI) foram Hymenaea stigonocarpa (15,06\%), Allagoptera campestris (6,36\%), Diospyros hispida (6,16\%), Rapanea guianensis (6,046\%) e Piptocarpha rotundifolia (5,33\%) (Quadro 2).

\section{REFERÊNCIAS BIBLIOGRÁFICAS}

COLE, M. M. Cerrado, caatinga and pantanal: The distribution and origin of the savanna vegetation of Brazil. Geography Journal, v. 106, n. 2, p. 168-179, 1960.

CRONQUIST, A. The evolution and classification of flowering plants. New York: The New York Botanical Garden, 1988. 555 p.

EITEN,G. The cerrado vegetation of Brazil. Botanical Review, v. 38, n. 2, p. 201-341, 1972.

MUELLER-DOMBOIS, D.; ELLENBERG, H. Aims and methods of vegetation ecology. New York: John Wiley \& Sons, 1974. $547 \mathrm{p}$.

RATTER, J. A. Transitions between cerrado and forest vegetation in Brasil. In: FURLEY, P.A.; PROCTOR, J.; RATTER, J. A. (Eds.) Nature and dynamics of forestsavanna boundaries. London: Chapman \& Hall, 1992. p. 51-76.

RATTER, J. A.; RIBEIRO, J. F.; BRIDGEWATER, S. Woody flora distribution of the cerrado biome: phytogeography and conservation priorities. In: CAVALCANTI, T. B. et al. (Org). CONGRESSO NACIONAL DE BOTÂNICA, 51, 2000, Brasília. Embrapa Recursos Genéticos e Biotecnologia, Sociedade Botânica do Brasil. 2000. p. 340-342.

ROYAL BOTANIC GARDENS OF KEW. Index Kewensis on compact disc - Manual. Oxford: Oxford University Press, 1997. 67 p. 
Q u adro 1 - E spécies lenhosas em cerrado sensu stricto do Parque N acional da Serra do C ịpó $T$ able $1-W$ oody species in a stricto sen su, cerrado Serra do C ipó $N$ ational Park

\begin{tabular}{|c|c|}
\hline Annonaceae & $\begin{array}{l}\text { 1. Annona coriacea Mart. } \\
\text { 2. Annona crassiflora Mart. }\end{array}$ \\
\hline Apocynaceae & 3. Aspidosperma tomentosum Mart. \\
\hline Araliaceae & 4. Didymopanax macrocarpum Seem. \\
\hline Arecaceae & 5. Allagoptera campestris Kuntze \\
\hline Asteraceae & $\begin{array}{l}\text { 6. Piptocarpha rotundifolia (Less.) Baker } \\
\text { 7. Vanillosmopsis erythropappa } \text { Sch. Bip. }\end{array}$ \\
\hline Bignoniaceae & 8. Tabebuia ochracea (Cham.) Standl. \\
\hline Leguminosae Caesalpinioideae & $\begin{array}{l}\text { 9. Acosmium dasycarpum (Vogel) Yakovlev } \\
\text { 10. Bauhinia bongardii Steud. } \\
\text { 11. Hymenaea stigonocarpa Mart. }\end{array}$ \\
\hline Chrysobalanaceae & 12. Couepia grandiflora (Mart. \& Zucc.) Benth. ex Hook. f. \\
\hline Guttiferae & $\begin{array}{l}\text { 13. Kielmeyera coriacea (Spreng.) Mart. } \\
\text { 14. Kielmeyera petiolaris Mart. } \\
\text { 15. Kielmeyera variabilis Mart. }\end{array}$ \\
\hline Connaraceae & 16. Rourea induta Planch. \\
\hline Dilleniaceae & 17. Davilla rugosa Poir \\
\hline Ebenaceae & 18. Diospyros hispida A. DC. \\
\hline Erythroxylaceae & $\begin{array}{l}\text { 19. Erythroxylum daphnites Mart. } \\
\text { 20. Erythroxylum tortuosum Mart. }\end{array}$ \\
\hline Leguminosae Papilionoideae & $\begin{array}{l}\text { 21. Dalbergia miscolobium Benth. } \\
\text { 22. Machaerium opacum Vogel }\end{array}$ \\
\hline Lamiaceae & 23. Hyptis cana Pohl ex Benth. \\
\hline Lythraceae & 24. Lafoensia pacari A. St.-Hil. \\
\hline Malpighiaceae & 25. Byrsonima verbascifolia Rich. ex A. Juss. \\
\hline Leguminosae Mimosoideae & $\begin{array}{l}\text { 26. Enterolobium gummiferum (Mart.) Macbr. } \\
\text { 27. Plathymenia reticulata Benth. } \\
\text { 28. Stryphnodendron adstringens (Mart.) Coville }\end{array}$ \\
\hline Myrsinaceae & 29. Rapanea guianensis Aubl. \\
\hline Myrtaceae & $\begin{array}{l}\text { 30. Eugenia aurata } \mathrm{Berg} \\
\text { 31. Myrcia lingua }(\mathrm{Berg}) \text { Mattos \& D. Legrand }\end{array}$ \\
\hline Nyctaginaceae & $\begin{array}{l}\text { 32. Guapira ferruginea (Klotzsch ex Choisy) Lundell } \\
\text { 33. Guapira noxia (Netto) Lundell }\end{array}$ \\
\hline Ochnaceae & 34. Ouratea spectabilis (Mart.) Engl. \\
\hline Opiliaceae & 35. Agonandra brasiliensis Benth. \& Hook. \\
\hline Proteaceae & 36. Roupala montana Aubl. \\
\hline Rubiaceae & $\begin{array}{l}\text { 37. Rudgea viburnoides (Cham.) Benth. } \\
\text { 38. Tocoyena formosa } \mathrm{K} \text {. Schum. }\end{array}$ \\
\hline Sapotaceae & $\begin{array}{l}\text { 39. Pouteria ramiflora (Mart.) Radlk. } \\
\text { 40. Pouteria torta (Mart.) Radlk. }\end{array}$ \\
\hline Solanaceae & 41. Solanum lycocarpum A. St.-Hil. \\
\hline Verbenaceae & 42. Lippia sp. \\
\hline Vochysiaceae & $\begin{array}{l}\text { 43. Qualea cordata Spreng. } \\
\text { 44. Qualea multiflora Mart. }\end{array}$ \\
\hline
\end{tabular}


Q uadro 2 - Parâm etros fitossociológicos em cerrado no Parque N acional da Serra do C ipó: D A - densidade absoluta; D R densidade relativa; FA - freqüência absoluta; FR - freqüência relativa; D oA - dom inância absoluta; DoR - dom inância relativa e V I - valor de im portância

$T a b l e ~ 2$ - Phytosociological param eters of cerrado in Serra do Cipó $N$ ational Park: D A - absolute dom inance; $D R$ - relative dom inance; FA - absolute frequency; $F R$-relative frequency; $D$ oA - absolute dom inance; $D$ oR - relative dom inance; and VI - im portance value

\begin{tabular}{|c|c|c|c|c|c|c|c|}
\hline Espécie & $\mathrm{DA}$ & DR & FA & FR & DoA & DoR & VI \\
\hline Hymenaea stigonocarpa & 494,4 & 16,60 & 83,33 & 5,35 & 3,4636 & 23,24 & 45,20 \\
\hline Allagoptera campestris & 250,0 & 8,40 & 83,33 & 5,35 & 0,7958 & 5,34 & 19,08 \\
\hline Diospyros hispida & 222,2 & 7,46 & 100,00 & 6,42 & 0,6883 & 4,62 & 18,50 \\
\hline Rapanea guianensis & 172,2 & 5,78 & 66,67 & 4,28 & 1,2043 & 8,08 & 18,14 \\
\hline Piptocarpha rotundifolia & 155,6 & 5,22 & 83,33 & 5,35 & 0,8098 & 5,43 & 16,01 \\
\hline Aspidosperma tomentosum & 144,4 & 4,85 & 83,33 & 5,35 & 0,7075 & 4,75 & 14,95 \\
\hline Erythroxylum daphnites & 172,2 & 5,78 & 66,67 & 4,28 & 0,3789 & 2,54 & 12,60 \\
\hline Acosmium dasycarpum & 111,1 & 3,73 & 83,33 & 5,35 & 0,4264 & 2,86 & 11,94 \\
\hline Byrsonima verbascifolia & 77,8 & 2,61 & 91,67 & 5,88 & 0,4047 & 2,72 & 11,21 \\
\hline Pouteria ramiflora & 116,7 & 3,92 & 75,00 & 4,81 & 0,3664 & 2,46 & 11,19 \\
\hline Kielmeyera coriacea & 111,1 & 3,73 & 75,00 & 4,81 & 0,2066 & 1,39 & 9,93 \\
\hline Guapira noxia & 50,0 & 1,68 & 33,33 & 2,14 & 0,9077 & 6,09 & 9,91 \\
\hline Annona coriacea & 66,7 & 2,24 & 25,00 & 1,60 & 0,8443 & 5,67 & 9,51 \\
\hline Stryphnodendron adstringens & 83,3 & 2,80 & 58,33 & 3,74 & 0,3486 & 2,34 & 8,88 \\
\hline Agonandra brasiliensis & 94,4 & 3,17 & 41,67 & 2,67 & 0,2734 & 1,83 & 7,68 \\
\hline Pouteria torta & 22,2 & 0,75 & 16,67 & 1,07 & 0,8545 & 5,73 & 7,55 \\
\hline Ouratea spectabilis & 83,3 & 2,80 & 50,00 & 3,21 & 0,1737 & 1,17 & 7,17 \\
\hline Hyptis cana & 66,7 & 2,24 & 33,33 & 2,14 & 0,2400 & 1,61 & 5,99 \\
\hline Roupala montana & 61,1 & 2,05 & 33,33 & 2,14 & 0,1420 & 0,95 & 5,14 \\
\hline Enterolobium gummiferum & 38,9 & 1,31 & 25,00 & 1,60 & 0,3095 & 2,08 & 4,99 \\
\hline Qualea multiflora & 66,7 & 2,24 & 8,33 & 0,53 & 0,1681 & 1,13 & 3,90 \\
\hline Tocoyena formosa & 33,3 & 1,12 & 33,33 & 2,14 & 0,0944 & 0,63 & 3,89 \\
\hline Dalbergia miscolobium & 22,2 & 0,75 & 33,33 & 2,14 & 0,1355 & 0,91 & 3,79 \\
\hline Myrcia lingua & 22,2 & 0,75 & 33,33 & 2,14 & 0,0517 & 0,35 & 3,23 \\
\hline Annona crassiflora & 11,1 & 0,37 & 16,67 & 1,07 & 0,2157 & 1,45 & 2,89 \\
\hline Erythroxylum tortuosum & 22,2 & 0,75 & 25,00 & 1,60 & 0,0401 & 0,27 & 2,62 \\
\hline Machaerium opacum & 16,7 & 0,56 & 16,67 & 1,07 & 0,1097 & 0,74 & 2,37 \\
\hline Kielmeyera variabilis & 22,2 & 0,75 & 16,67 & 1,07 & 0,0510 & 0,34 & 2,16 \\
\hline Solanum lycocarpum & 11,1 & 0,37 & 16,67 & 1,07 & 0,0975 & 0,65 & 2,10 \\
\hline Couepia grandiflora & 22,2 & 0,75 & 16,67 & 1,07 & 0,0340 & 0,23 & 2,04 \\
\hline Plathymenia reticulata & 27,8 & 0,93 & 8,33 & 0,53 & 0,0830 & 0,56 & 2,02 \\
\hline Vanillosmopsis erythropappa & 11,1 & 0,37 & 16,67 & 1,07 & 0,0560 & 0,38 & 1,82 \\
\hline Tabebuia ochracea & 11,1 & 0,37 & 16,67 & 1,07 & 0,0188 & 0,13 & 1,57 \\
\hline Eugenia aurata & 11,1 & 0,37 & 8,33 & 0,53 & 0,0803 & 0,54 & 1,45 \\
\hline Bauhinia bongardii & 16,7 & 0,56 & 8,33 & 0,53 & 0,0195 & 0,13 & 1,23 \\
\hline Lafoensia pacari & 11,1 & 0,37 & 8,33 & 0,53 & 0,0143 & 0,10 & 1,00 \\
\hline Kielmeyera petiolaris & 5,6 & 0,19 & 8,33 & 0,53 & 0,0299 & 0,20 & 0,92 \\
\hline Rudgea viburnoides & 5,6 & 0,19 & 8,33 & 0,53 & 0,0177 & 0,12 & 0,84 \\
\hline Davilla rugosa & 5,6 & 0,19 & 8,33 & 0,53 & 0,0128 & 0,09 & 0,81 \\
\hline Guapira ferruginea & 5,6 & 0,19 & 8,33 & 0,53 & 0,0075 & 0,05 & 0,77 \\
\hline Rourea induta & 5,6 & 0,19 & 8,33 & 0,53 & 0,0044 & 0,03 & 0,75 \\
\hline Qualea cordata & 5,6 & 0,19 & 8,33 & 0,53 & 0,0044 & 0,03 & 0,75 \\
\hline Lippia sp. & 5,6 & 0,19 & 8,33 & 0,53 & 0,0044 & 0,03 & 0,75 \\
\hline Didymopanax macrocarpum & 5,6 & 0,19 & 8,33 & 0,53 & 0,0044 & 0,03 & 0,75 \\
\hline
\end{tabular}

